\title{
The long term effect of metformin plus DPP-4 inhibitor switching from metformin plus pioglitazone combination therapy in Type 2 diabetes
}

\author{
Yonghyun Kim, Donghyun Shin \\ Endocrinology Dept., Internal Hedicine \\ Bundang Jesaeng Hospital, Seongnam, S. Korea
}

\section{Background}

- Two years ago, we presented the short term 6 month results of metformin plus DPP-4 inhibitor combination therapy when we can't reach the target below $7 \%$ of $\mathrm{HgA1}$ c with metformin plus pioglitazone combination that is best in terms of relieving insulin resistance in early diabetes,

- Switching the pioglitazone to the DPP-4 inhibitor that improves insulin secretory dysfunction can be the next useful step to attain glucose control goal.

- DPP-4 inhibitors that increase insulin secretion by glucose dependent manner can also relieve insulin resistance because they improve first phase insulin secretion defect and prevent late hyperinsulinemia.

- DPP-4 inhibitor is also better than pioglitazone in weight aspect.

\section{Method}

- Total 111 patients were followed by 26.5 month $( \pm 9.7)$ after switching from metformin plus pioglitazone.

- $15 \mathrm{mg}$ of pioglitazone that is usual dosage in this country was switched to DPP-4 inhibitors without changing the dose of metformin.

- The change of medication during follow up was examined.

- HgA1c level, insulin resistance index HOMA-IR, weight change before and after switching was compared in patients who maintained metformin plus first dose of DPP-4 inhibitor.

\section{Result}

- Sulfonylurea was added in 6\% (7/111) and pioglitazone was added again in 7\% $(7 / 111)$ for adequate glucose control.

- The dose of DPP-4 inhibitor was decreased in $6 \%(7 / 111)$ during the follow up period.

- Metformin plus first dose of DPP-4 inhibitor was maintained in 80\% ( $89 / 111)$.

- The dose of metformin was increased in $41 \%$ ( 40/89) and unchanged in 42\% (41/89) and decreased in $7 \%$.

- The mean dose of metformin to maintain HgA1c target was elevated by $260 \mathrm{mg}$ in the end.

- HgA1c was improved in $76 \%$ ( $68 / 89$ ) from $7.40 \%$ to $6.66 \%$ and the metformin dose was increased by 240 mg. HgA1c was aggravated in $22 \%$ ( $20 / 89$ ) from $6.85 \%$ to $7.22 \%$.

- HOMA-IR was improved in 46\% ( $0.91 \pm 0.92)$ and aggravated in 53\% (-1.25 \pm 1.17$)$ Mean HOMA-IR change was $-0.24 \pm 1.51$.

- Mean weight was reduced by $2.23 \mathrm{~kg}( \pm 3.17)$ as a whole.

The weight was decreased in $72 \%(3.63 \pm 2.51)$ and increased in $19 \%(1.77 \pm 1.73)$.

\section{Conclusion}

Although the dose of metformin was slightly increased to maintain glucose control target with time,

metformin plus DPP-4 inhibitor combination can be a good treatment option

in controlling blood glucose \&

maintaining insulin resistance \&

as compared to metformin plus pioglitazone combination. 